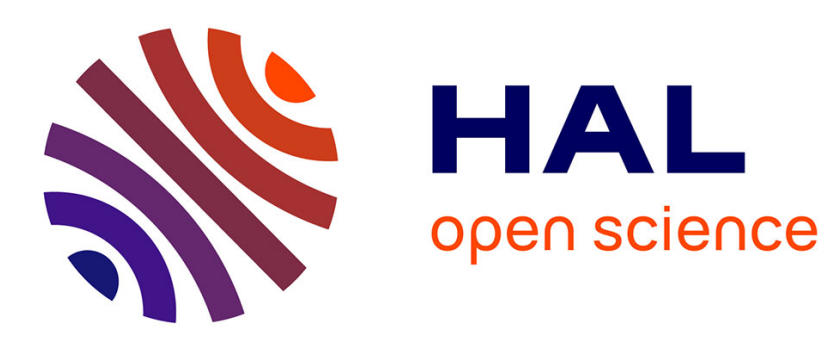

\title{
Numerical Minimization of Dirichlet Laplacian Eigenvalues of Four-Dimensional Geometries
}

\author{
Pedro Antunes, Edouard Oudet
}

\section{To cite this version:}

Pedro Antunes, Edouard Oudet. Numerical Minimization of Dirichlet Laplacian Eigenvalues of Four-Dimensional Geometries. SIAM Journal on Scientific Computing, 2017, 39 (3), pp.B508-B521. 10.1137/16M1083773 . hal-02009467

\section{HAL Id: hal-02009467 https://hal.univ-grenoble-alpes.fr/hal-02009467}

Submitted on 6 Feb 2019

HAL is a multi-disciplinary open access archive for the deposit and dissemination of scientific research documents, whether they are published or not. The documents may come from teaching and research institutions in France or abroad, or from public or private research centers.
L'archive ouverte pluridisciplinaire HAL, est destinée au dépôt et à la diffusion de documents scientifiques de niveau recherche, publiés ou non, émanant des établissements d'enseignement et de recherche français ou étrangers, des laboratoires publics ou privés. 


\title{
NUMERICAL MINIMIZATION OF DIRICHLET-LAPLACIAN EIGENVALUES OF FOUR-DIMENSIONAL GEOMETRIES
}

\author{
PEDRO R.S. ANTUNES AND ÉDOUARD OUDET
}

\begin{abstract}
We develop the first numerical study in four dimension of optimal eigenmodes associated to the Dirichlet Laplacian. We describe an extension of the Method of Fundamental Solutions adapted to the four dimensional context. Based on our numerical simulation and a post processing adapted to the identification of relevant symmetries, we provide and discuss the numerical description of the $8^{\text {th }}$ first optimal domains.
\end{abstract}

\section{INTRODUCTION}

Shape and design optimization problems involving eigenvalues of elliptic operators is a very active topic in spectral theory and arises in many physical applications. A better understanding of this questions allows for example to tune and improve the acoustic properties of musical instruments $[8,19,30,40,42]$ or to find optimal designs of composite materials (eg. [2]). A typical situation in engineering is the identification of shapes or designs of structures to prescribe their mechanical properties: being either as rigid or as soft as possible (e.g. [29]), avoiding torsional oscillations (e.g. [11]), etc. Such problems appear also in the context of electromagnetism when considering the design of optimal accelerator cavities (eg. [1]).

A prototype of these shape optimization problems is the minimization of Dirichlet eigenvalues of the Laplacian, with a volume constraint. Whereas classical and widely studied, this problem collects all the main computational difficulties of design optimization: non-smoothness, local minima, high number of degrees of freedom and a computationally expansive cost function.

Let $\Omega \subset \mathbb{R}^{d}$ be a bounded open set, not necessarily connected, and consider the Dirichlet eigenvalue problem,

$$
\begin{cases}-\Delta u=\lambda u & \text { in } \Omega \\ u=0 & \text { on } \partial \Omega\end{cases}
$$

defined in the Sobolev space $H_{0}^{1}(\Omega)$. We will denote the eigenvalues by $0<\lambda_{1}(\Omega) \leq$ $\lambda_{2}(\Omega) \leq \ldots$ where each $\lambda_{k}(\Omega)$ is counted with its multiplicity and the corresponding orthonormal real eigenfunctions by $u_{i}, i=1,2, \ldots$. The shape optimization can be

Date: September 21, 2016.

Key words and phrases. Dirichlet Laplacian, eigenvalues, higher dimensional space, shape optimization.

P.A. was partially supported by FCT, Portugal, through the program "Investigador FCT" with reference IF/00177/2013 and the scientific project PTDC/MAT- CAL/4334/2014. Édouard Oudet gratefully acknowledges the support of the ANR, through the projects COMEDIC, PGMO and OPTIFORM.. 
formulated as determining

$$
\lambda_{k}^{\star}=\operatorname{Min}\left\{\lambda_{k}(\Omega): \Omega \subset \mathbb{R}^{d},|\Omega|=1\right\}, k=1,2, \ldots
$$

The existence of minimizing domains among quasi-open sets was recently proved (cf. $[14,37]$ ) and some numerical studies allowed to suggest candidates to be minimizers at the beginning of the spectrum and also to explore some properties such as connectedness, symmetry and multiplicity of optimal eigenvalues (eg. [41, 6, 43]).

In this paper we address the solution of shape optimization problems for the Dirichlet eigenvalues of the Laplacian of four-dimensional geometries. Besides the fact that four-dimensional differential geometry may present interesting features (eg. [21, 26, 48]), also the solution of $4 \mathrm{D}$ shape optimization problems and the calculation of eigenvalues of $4 \mathrm{D}$ geometries are challenging from the computational point of view. Indeed, to the best of our knowledge this is the first time that a $4 \mathrm{D}$ shape optimization related with eigenvalues is considered. For this purpose we used the Method of Fundamental Solutions (MFS) as solver for the eigenvalue problem. The MFS is a meshfree method and thus, in this context, it avoids meshing 4D domains which could be a difficult and time consuming task. Moreover, the huge dimension of the matrices associated to classical mesh-type methods such as finite element methods would probably be prohibitive for solving the eigenvalue problem accurately in a reasonable time. On the other hand, the problem can be circumvented using spectral methods, such as the MFS. We can prove that the solution of the eigenvalue problem is also solution of an integral equation defined on the boundary of a domain which can be seen as a space dimension reduction and the problem of solving an eigenvalue problem with $4 \mathrm{D}$ domain is replaced by the solution of an integral equation defined on a 3D hyper-surface.

The convergence of the MFS, when applied to smooth shapes is known to be very fast, in some cases even exponential (cf. [33, 4, 10]), which ensures that this integral equation can be solved accurately with relatively small dimension matrices.

The plan of the paper is the following. In section 2 we give a brief description of some aspects of the numerical approach, namely we define a parameterization of a general star-shaped domain in terms of 4D Hyper-spherical Harmonics, we present an algorithm for the distribution of the collocation points and source-points for the MFS and use Betcke-Trefethen subspace angle approach for the eigenvalue calculation and describe an algorithm for the optimization. Section 3 describes an algorithm for the visualization of the optimal shapes and present some of the numerical results that we gathered. In Section 4 we discuss some of the results that we obtained.

\section{NumERICAL SOLUTION OF THE SHAPE OPTIMIZATION PROBLEM}

2.1. Brief description of the MFS. We will use the MFS as forward solver for the eigenvalue problem. We take a fundamental solution of the Helmholtz equation in $\mathbb{R}^{d}$,

$$
\Phi_{\lambda}(x)=\frac{i}{4}\left(\frac{\sqrt{\lambda}}{2 \pi|x|}\right)^{\frac{d-2}{d}} H_{\frac{d-2}{2}}^{(1)}(\sqrt{\lambda}|x|),
$$


where $|\cdot|$ denotes the Euclidean norm in $\mathbb{R}^{d}$ and $H_{\frac{d-2}{2}}^{(1)}$ is a Hänkel function defined by Bessel functions (e.g. [38]),

$$
H_{\frac{d-2}{2}}^{(1)}(\cdot)=J_{\frac{d-2}{2}}(\cdot)+i Y_{\frac{d-2}{2}}(\cdot) .
$$

The MFS approximation is a linear combination

$$
u \approx \tilde{u}(x)=\sum_{i=1}^{M} \beta_{i} \phi_{j}(x)
$$

where

$$
\phi_{j}=\Phi_{\lambda}\left(\cdot-y_{j}\right)
$$

are $M$ point sources centered at some points $y_{j}$ that are placed on an admissible source set $\hat{\Gamma}$ which does not intersect $\bar{\Omega}$. By construction, the MFS approximation (4) satisfies the PDE of the eigenvalue problem (1) and the numerical accuracy of the solution is related with the accuracy of the approximation of the boundary condition. As proven in [5], given an admissible source set $\hat{\Gamma}$, which can be a Jordan curve surrounding $\partial \Omega$, we have the following result,

Theorem 1. If $\hat{\Gamma}$ is an admissible source set, then

$$
S(\hat{\Gamma})=\operatorname{span}\left\{\left.\Phi_{\lambda}(\cdot-y)\right|_{\Omega}: y \in \hat{\Gamma}\right\}
$$

is dense in $\mathcal{H}_{\lambda}(\Omega)=\left\{v \in H^{1}(\Omega):(\Delta+\lambda) v=0\right\}$, with the $H^{1}(\Omega)$ topology.

By the Sobolev regularity theorem (cf. [31]), assuming that $\Omega$ is $C^{1,2}$, we know that the eigenfunctions belong to the Sobolev space $H^{2}(\Omega)$ and thus, the density theorem 1 states that an eigenfunction can be arbitrarily well approximated by a MFS linear combination (4).

2.2. Parametrization of domains. We will restrict the shape optimization (2) to a similar problem defined in the class of sets that are finite unions of star-shaped domains. This restriction is only related with the simplicity of parameterizing a generic star-shaped domain and not to limitations of the MFS which can also be applied for non-simply connected domains (cf. [16]). Moreover, using Wolf-Keller theorem, if some optimizer for a given eigenvalue $\lambda_{k}$ is a finite union of star-shaped domains, then each of its connected components is a minimizer for a previous eigenvalue. Thus, we can restrict our attention to star-shaped domains and study the disconnected case using Wolf-Keller result ([49]).

A $4 \mathrm{D}$ star-shaped domain is isometric to the domain whose boundary is parameterized by

$$
\left\{\begin{array}{l}
x=r(\beta, \theta, \phi) \sin (\beta) \sin (\theta) \cos (\phi) \\
y=r(\beta, \theta, \phi) \sin (\beta) \sin (\theta) \sin (\phi) \\
z=r(\beta, \theta, \phi) \sin (\beta) \cos (\theta) \\
w=r(\beta, \theta, \phi) \cos (\beta)
\end{array}\right.
$$

where $r(\beta, \theta, \phi)>0$, for $\beta \in[0, \pi], \theta \in[0, \pi]$ and $\phi \in[0,2 \pi[$.

The Laplace-Beltrami operator on $S^{3}$ is defined by

$$
\Delta_{S^{3}}=\frac{1}{\sin ^{2} \beta} \frac{\partial}{\partial \beta} \sin ^{2} \beta \frac{\partial}{\partial \beta}+\frac{1}{\sin ^{2} \beta} \Delta_{S^{2}},
$$


where $\Delta_{S^{2}}$ is the Laplace-Beltrami operator on the unitary sphere $S^{2}$. We define the family of 4D Hyper-spherical Harmonics (4D HSH), by

$$
\begin{aligned}
& n=0,1,2, \ldots \quad \beta \in[0, \pi] \\
& S_{n l}^{m}(\beta, \theta, \phi)=c_{n, l, m} \sin ^{l}(\beta) C_{n-l}^{l+1}(\cos (\beta)) Y_{l}^{m}(\theta, \phi)(\beta, \theta, \phi), \quad 0 \leq l \leq n \quad, \quad \theta \in[0, \pi] \quad, \\
& -l \leq m \leq l \quad \phi \in[0,2 \pi[
\end{aligned}
$$

where $Y_{l}^{m}$ are 3D spherical harmonics, $C_{n-1}^{l+1}$ are Gegenbauer polynomials and

$$
c_{n, l, m}=2^{l+\frac{1}{2}} \sqrt{\frac{(n+1) \Gamma(n-l+1)}{\pi \Gamma(n+l+2)}} \Gamma(l+1) .
$$

The 4D HSH are eigenfunctions of $\Delta_{S^{3}}$,

$$
\Delta_{S^{3}} S_{n l}^{m}=-l(l+2) S_{n l}^{m},
$$

and form an orthonormal basis defined on the hyper-sphere, satisfying the normalization condition

$$
\int_{0}^{2 \pi} \int_{0}^{\pi} \int_{0}^{\pi} S_{n l}^{m}(\beta, \theta, \phi) S_{n^{\prime} l^{\prime}}^{m^{\prime}}(\beta, \theta, \phi) \sin ^{2} \beta \sin \theta d \beta d \theta d \phi=\delta_{n, n^{\prime}} \delta_{l, l^{\prime}} \delta_{m, m^{\prime}} .
$$

Thus, the function $r$ in (6), which maps the unitary hyper-sphere $S^{3}$ to the boundary of a generic domain will be approximated by the expansion,

$$
r(\beta, \theta, \phi) \approx r_{N}(\beta, \theta, \phi)=\sum_{n=0}^{N} \sum_{l=0}^{n} \sum_{m=-l}^{l} \alpha_{n, l, m} S_{n l}^{m}(\beta, \theta, \phi) .
$$

We define the vector $\mathcal{V} \in \mathbb{R}^{P}$, where $P=\frac{1}{6}(N+1)(N+2)(2 N+3)$, containing all the coefficients $\alpha_{n, l, m}$ and the $4 \mathrm{D}$ shape optimization procedure will be performed by searching for optimal vectors $\mathcal{V}$. In this work we considered $N=10$, which implies that the vector $\mathcal{V}$ has 506 components.

2.3. Generation of points for the MFS. As mentioned in section 2.1, the MFS approximation (4) satisfies the PDE of the problem, and thus we can focus on the approximation of the boundary condition. We will consider a discrete set of collocation points on the boundary where we will impose the boundary conditions of the problem. Thus, we need to have an algorithm for distributing points on the boundary of a $4 \mathrm{D}$ domain with boundary defined by (6). Our approach is to define an almost uniformly distributed set of points on the 4D unitary hyper-sphere and then map these points to the boundary of the domain taking into account the function $r$.

We start with a 3D unitary sphere parameterized by

$$
\left\{\begin{array}{l}
x=\sin (\theta) \cos (\phi) \\
y=\sin (\theta) \sin (\phi) \\
z=\cos (\theta)
\end{array}\right.
$$

with $\theta \in[0, \pi]$ and $\phi \in[0,2 \pi[$.

A naive choice could be to consider the points generated with equally spaced angles $\theta$ and $\phi$ but it is well known that this procedure does not produce a uniform distribution of points. Instead, we define an integer number $M_{\mathcal{C}}$ corresponding to the number of collocation points that we would like to place and try to have 
locally the same variation of the angles $\theta$ and $\phi$ and that the product of these two quantities is approximately equal to the average area of each point, i.e.

$$
\Delta \theta \approx \Delta \phi, \quad \Delta \theta \Delta \phi \approx \frac{4 \pi}{M_{\mathcal{C}}} \Rightarrow \Delta \theta \approx \Delta \phi \approx \sqrt{\frac{4 \pi}{M_{\mathcal{C}}}} .
$$

Next, we fix a meridian, for example corresponding to $\phi=0$ whose length is $\pi$. Thus, in order to have (10), the number of divisions along this meridian is equal to $\left\lceil\sqrt{\frac{M_{\mathcal{C}} \pi}{4}}\right\rceil$ (Figure 1-left). Finally, each of the divisions on the meridian corresponds to a parallel defined by $\theta=\theta_{i}$. This parallel is a circumference of radius $\sin \left(\theta_{i}\right)$ and, thus has perimeter equal to $2 \pi \sin \left(\theta_{i}\right)$. Again, in order to have (12) we must place $\left\lceil\sqrt{\frac{M_{\mathcal{C}} \pi}{4}} \sin \left(\theta_{i}\right)\right\rceil$ points uniformly distributed on the parallel (Figure 1-right). In Figure 2 we plot the points generated by our algorithm with $M_{\mathcal{C}}=500$ (left)
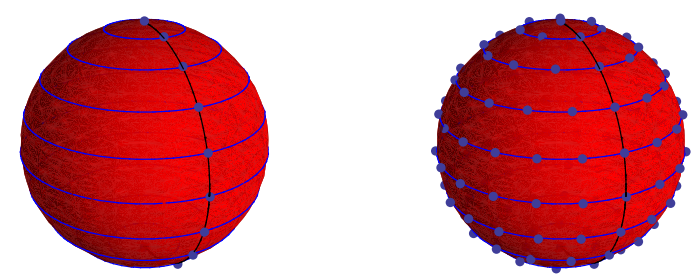

Figure 1. Algorithm for distributing points on a 3D sphere.

and $M_{\mathcal{C}}=2000$ (right). The extension of this algorithm for the $4 \mathrm{D}$ sphere is
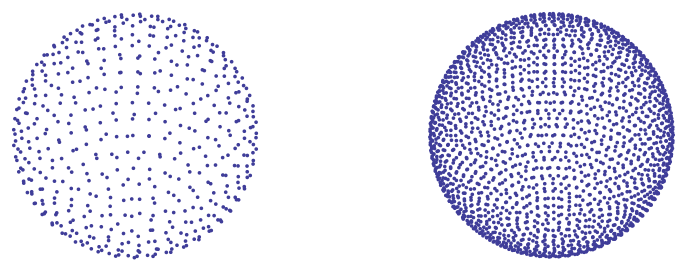

Figure 2. Points on the sphere generated by our algorithm with $M_{\mathcal{C}}=500$ (left) and $M_{\mathcal{C}}=2000$ (right).

straightforward. We take the 4D unitary sphere,

$$
\left\{\begin{array}{l}
x=\sin (\beta) \sin (\theta) \cos (\phi) \\
y=\sin (\beta) \sin (\theta) \sin (\phi) \\
z=\sin (\beta) \cos (\theta) \\
w=\cos (\beta)
\end{array}\right.
$$


with $\beta \in[0, \pi], \theta \in[0, \pi]$ and $\phi \in[0,2 \pi[$. Again, we try to have locally the same variation of the three angles and that the product of these two quantities is approximately equal to the average area of each point, using the fact that the surface area of a $4 \mathrm{D}$ unitary sphere is equal to $2 \pi^{2}$,

$$
\Delta \theta \approx \Delta \phi \approx \Delta \beta, \quad \Delta \theta \Delta \phi \Delta \beta \approx \frac{2 \pi^{2}}{M_{\mathcal{C}}} \Rightarrow \Delta \theta \approx \Delta \phi \approx \Delta \beta \approx \sqrt[3]{\frac{2 \pi^{2}}{M_{\mathcal{C}}}}:=C .
$$

Now, the number of $4 D$ parallels corresponding to division along the angle $\beta$ is equal to $\left\lceil\frac{\pi}{C}\right\rceil$, and each of these $4 D$ parallels defined by $\beta=\beta_{i}$ are $3 \mathrm{D}$ spheres of radius $\sin \left(\beta_{i}\right)$. Therefore, we can apply the previous algorithm for $3 \mathrm{D}$ spheres. The surface area of a $3 \mathrm{D}$ sphere of radius $R$ is given by $4 \pi R^{2}$, and, thus, we use the previous algorithm to place $\left\lceil\frac{4 \pi \sin ^{2}\left(\beta_{i}\right)}{C}\right\rceil$ points on each of the $4 D$ parallels.

This distribution of points on the sphere is not optimal and other possibilities could be considered. Indeed, the problem of distributing $N$ points on the sphere in such a way that we maximize the minimum distance between any pair of points is a classical problem known as Tammes problem $([46,17])$. The problem was already solved for some particular small numbers $N$ (eg. [25, 20, 39]). Another possible criteria for determining optimal locations for the nodes is to calculate the Fekete points $x_{i}, i=1, \ldots, N$ that minimize the energy,

$$
E(N)=\sum_{1 \leq i<j \leq N} \frac{1}{\left|x_{i}-x_{j}\right|} .
$$

In this case, the point $x_{i}$ correspond to charged particles which repel each other according to Coulomb's law. Several works addressed the numerical solution of this problem for an arbitrary number $N$, even for high dimensional hyper-sphere $([22,27,47])$. Other approaches for the distribution of nodes could be considered. For example in [7] it was described an algorithm for distributing nodes on the boundary of $3 \mathrm{D}$ shapes. However, from the computational point of view, all these approaches are more expensive than the algorithm that was described, which allows to obtain good distributions of arbitrary number of nodes in less than one second.

In the context of the application of MFS, the most important issue to have highly accurate results is the choice for the location of the source points $y_{j}$ (eg. $[4,3,10,7]$ ). In this work we will follow the choice proposed in [4, 7]. We take $M_{\mathcal{C}}$ collocation points $x_{i}, i=1, \ldots, M_{\mathcal{C}}$ on the boundary of the domain and for each of these points we calculate the outward unitary vector $n_{i}$, which is normal to the boundary at $x_{i}$. The source points are defined by

$$
y_{i}=x_{i}+\delta n_{i},
$$

where $\delta$ is a parameter chosen such that the source points remain outside $\bar{\Omega}$. The numerical results that we will present in Section 3 were obtained with $M_{\mathcal{C}} \approx 7000$ and $\delta \approx 0.2$.

2.4. Eigenvalue calculation. The MFS approximation satisfies the PDE of the eigenvalue problem and thus the approximation for an eigenvalue is determined searching for the values $\lambda$ such that there exists a (non zero) MFS function $\tilde{u}$ fitting the null boundary conditions. We used Betcke-Trefethen subspace angle approach for the eigenvalue calculation (cf. [13]). We distribute randomly some points $z_{i} \in \Omega, \mathrm{i}=1, \ldots, M_{\mathcal{I}}$, define the matrices $\mathbf{A}_{B}(\lambda), \mathbf{A}_{I}(\lambda)$, where

$$
\left[\mathbf{A}_{B}(\lambda)\right]_{i, j}=\Phi_{\lambda}\left(x_{i}-y_{j}\right) \quad\left[\mathbf{A}_{I}(\lambda)\right]_{i, j}=\Phi_{\lambda}\left(z_{i}-y_{j}\right)
$$


and

$$
\mathbf{A}(\lambda):=\left[\begin{array}{c}
\mathbf{A}_{B}(\lambda) \\
\mathbf{A}_{I}(\lambda)
\end{array}\right]
$$

and calculate the $\mathbf{Q R}$ decomposition of the matrix $\mathbf{A}(\lambda)$,

$$
\left[\begin{array}{l}
\mathbf{A}_{B}(\lambda) \\
\mathbf{A}_{I}(\lambda)
\end{array}\right]=\left[\begin{array}{l}
\mathbf{Q}_{B}(\lambda) \\
\mathbf{Q}_{I}(\lambda)
\end{array}\right] \mathbf{R}(\lambda) .
$$

Then, we study the evolution of the eigenvalue of $\mathbf{Q}_{\mathbf{B}}(\lambda)$ with smallest magnitude, which will be called $\sigma(\lambda)$. The approximations for the eigenvalues of the DirichletLaplacian are the local minima of $\sigma(\lambda)$ (cf. [13]). In Figure 3 we plot $\sigma(\lambda)$ for $\lambda \in[5,10]$ obtained for the $4 \mathrm{D}$ ball with unit volume and we can locate three eigenvalues in this interval. The method is not much sensitive to the choice of interior points $M_{\mathcal{I}}$ and in all the numerical simulations we fixed $M_{\mathcal{I}}=50$. In

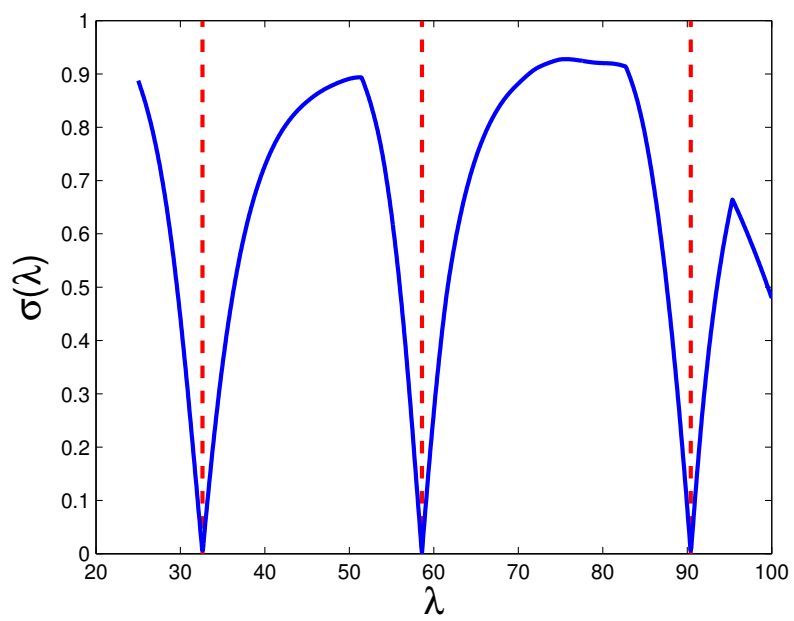

Figure 3. Plot of $\sigma(\lambda)$ for the $4 \mathrm{D}$ ball with unit volume.

Figure 4 we show some results for the convergence to the first eigenvalue, as a function of the number of collocation points $M_{\mathcal{C}}$, and three different choices of $\delta$. We can observe that the method has fast convergence and with $M_{\mathcal{C}}=3500$ we obtain relative errors close to machine level precision. Moreover, the best results are obtained for larger parameter $\delta$, which typically happens for the ball (eg. $[4,10,7])$.

2.5. Optimization algorithm. A very useful mathematical tool for solving shape optimization problems involving Dirichlet eigenvalues of the Laplacian is the Hadamard formula of derivation with respect to the domain (eg. [31]). Consider an application $\Psi(t)$ such that $\Psi: t \in\left[0, T\left[\rightarrow W^{1, \infty}\left(\mathbb{R}^{d}, \mathbb{R}^{d}\right)\right.\right.$ is differentiable at 0 with $\Psi(0)=I, \Psi^{\prime}(0)=V$, where $W^{1, \infty}\left(\mathbb{R}^{d}, \mathbb{R}^{d}\right)$ is the set of bounded Lipschitz maps from $\mathbb{R}^{d}$ into itself, $I$ is the identity and $V$ is a deformation field. Following the notation of [31], we will denote by $\Omega_{t}=\Psi(t)(\Omega), \lambda_{k}(t)=\lambda_{k}\left(\Omega_{t}\right)$ and by $u_{k}$ an associated normalized eigenfunction in $H_{0}^{1}(\Omega)$. The domains considered are smooth (see section 2.2), thus, if we assume that the eigenvalue $\lambda_{k}(\Omega)$ is simple then

$$
\lambda_{k}^{\prime}(0)=-\int_{\partial \Omega}\left|\nabla u_{k}\right|^{2} V \cdot n d \sigma .
$$




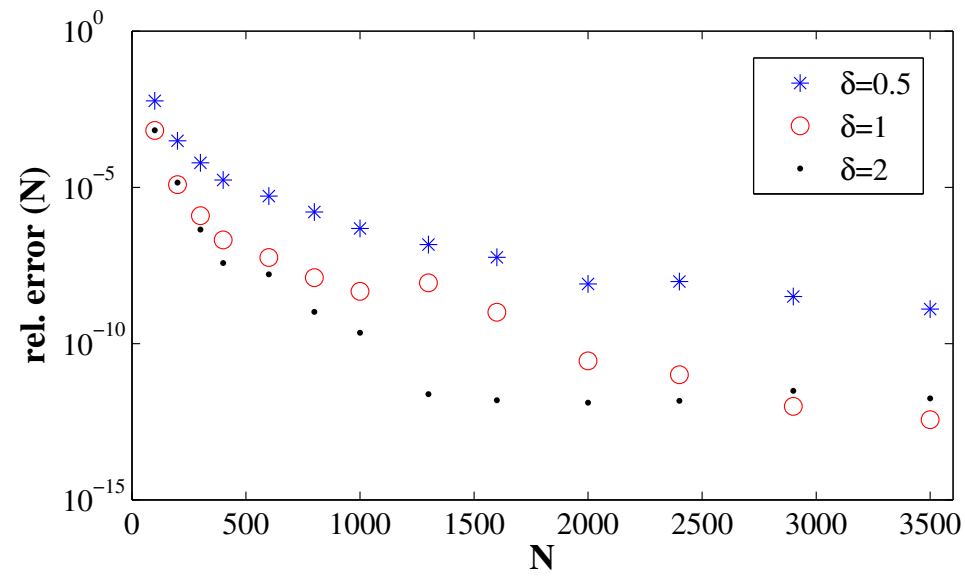

Figure 4. Convergence results for the first eigenvalue of the 4D ball.

We also know the derivative of the volume. Define the function $\operatorname{Vol}(t)=\left|\Omega_{t}\right|$, then, we have

$$
V o l^{\prime}(0)=\int_{\partial \Omega} V \cdot n d \sigma .
$$

Now we note that we know that given an homothety of ratio $t$, we have,

$$
\lambda_{k}(t \Omega)=\frac{\lambda_{k}(\Omega)}{t^{2}}
$$

and

$$
|t \Omega|=t^{4}|\Omega|,
$$

thus, instead of the optimization problem (2), we can study an equivalent problem which avoids the geometrical constraint

$$
\lambda_{k}^{\star}=\operatorname{Min}\left\{\lambda_{k}(\Omega)|\Omega|^{\frac{1}{2}}: \Omega \subset \mathbb{R}^{4}\right\}, k=1,2, \ldots
$$

It is well known that typically the minimizers correspond to domains for which the optimal eigenvalue is multiple and we must deal with the non-smoothness of the cost function,

$$
\mathcal{F}_{k}(\mathcal{V})=\lambda_{k}(\Omega)|\Omega|^{\frac{1}{2}},
$$

where $\Omega$ is obtained from $\mathcal{V}$, by using (6) and (8). Note that the directional derivatives of the eigenvalues with respect to a given perturbation field exist even in the case of multiple eigenvalues (eg. [31]). We will denote by $g_{k} \in \mathbb{R}^{P}$, the shape gradient of $\mathcal{F}_{k}$, that is a vector whose $i$-th component is the shape derivative of $\mathcal{F}_{k}$ with respect to the $i$-th component of the vector $\mathcal{V}$ and split the optimization procedure in two steps. At first stage, we apply the quasi-Newton method LBFGS to the minimization of $\mathcal{F}_{k}$ (eg. [36]). Once we are close to a multiple eigenvalue, that is $\mathcal{F}_{k}-\mathcal{F}_{k-1}$ is small enough, we switch the way we calculate a search direction for performing a line search. For a given (unitary) vector $v \in \mathbb{R}^{P}$ and small $t$ we have

$$
\mathcal{F}_{k}(\mathcal{V}+t v) \approx \mathcal{F}_{k}(\mathcal{V})+t\left(g_{k} \cdot v\right)
$$


where $\left(g_{k} \cdot v\right)$ is the directional derivative of $\mathcal{F}_{k}$ on the direction defined by $v$. In a similar fashion we have

$$
\mathcal{F}_{k-1}(\mathcal{V}+t v) \approx \mathcal{F}_{k-1}(\mathcal{V})+t\left(g_{k-1} \cdot v\right) .
$$

We want to decrease both $\mathcal{F}_{k}$ and $\mathcal{F}_{k-1}$, thus, $v$ must be such that $\left(g_{k} \cdot v\right)$ and $\left(g_{k-1} . v\right)$ and both negative and the corresponding absolute values are as large as possible. Thus, it is natural to define the search direction $v^{*}$ as the solution of the optimization problem

$$
\min _{v \in \mathbb{R}^{P}:\|v\|=1} \max \left(g_{k-1} \cdot v, g_{k} \cdot v\right) .
$$

The generalization for an eigenvalue with multiplicity $m$ is straightforward and instead of (16), the optimal search direction $v^{*}$ solves

$$
m^{*}=\min _{v \in \mathbb{R}^{P}:\|v\|=1} \max \left(g_{k-m+1} \cdot v, g_{k-m+2} . v, \ldots, g_{k} \cdot v\right)
$$

Another approach would be to use the formula for the derivative of a multiple eigenvalue (eg. $[28,45])$. The optimization procedure is summarized in Algorithm 1.

\section{NumericAl RESUlts}

Analyze geometrical properties of $4 \mathrm{D}$ shapes requires a special attention. In this spectral theory context, we focus on the identification of symmetries. Actually, the question of the existence of one or many symmetries of optimal profiles minimizing eigenmodes is one of the crucial issue in spectral optimization. This interest is motivated by the fact that symmetries are strongly connected to the multiplicity of eigenvalues in a non trivial way. We describe below a simple numerical approach that we developed to deduce symmetries of computed optimal shapes by algorithm 1. The following post processing is completely independant of previous optimization and could be used in a different context to identify orthogonal symmetries.

Due to the spherical parametrization (6), all the obtained profiles are star-shaped This fact makes the identification of symmetries easier: consider a sampling of the unite sphere $S^{3}$ associated to a finite collection of parameters $\left(\beta_{i}, \theta_{i}, \phi_{i}\right)$. For every optimal domain $\Omega$, we computed an analytic radius function $r_{\Omega}(\beta, \theta, \phi)$ which associates to $\left(\beta_{i}, \theta_{i}, \phi_{i}\right)$ by (6) a list of points $\left(P_{i}\right)$ of $\partial \Omega$. Actually, since $\Omega$ is starshaped, this map $X_{\Omega}$ from $S^{3}$ to $\partial \Omega$ defined by the system (6) is one to one. Consider now $s_{H}$ the symmetric transformation with respect to an hyperplane $H$. That is $s_{H}(x)=x+2 *\left(p_{H}(x)-x\right)$ where $p_{H}$ stands for the orthogonal projection on $H$. If $H$ is an hyperplane of symmetry of $\Omega$, we must have

$$
\left\|s_{H}\left(X_{\Omega}(\beta, \theta, \phi)\right)\right\|=r_{\Omega}\left(X_{\Omega}^{-1}\left(s_{H}\left(X_{\Omega}(\beta, \theta, \phi)\right)\right)\right) .
$$

We used previous equality to identify numerically the hyperplane of symmetries of a given shape. Actually, we define a simple cost function on the set of hyperplanes of $\mathbb{R}^{4}$, associated to the sampling $\left(\beta_{i}, \theta_{i}, \phi_{i}\right)$, by

$$
F(H)=\sum_{i}\left[\left\|s_{H}\left(X_{\Omega}\left(\beta_{i}, \theta_{i}, \phi_{i}\right)\right)\right\|-r_{\Omega}\left(X_{\Omega}^{-1}\left(s_{H}\left(X_{\Omega}\left(\beta_{i}, \theta_{i}, \phi_{i}\right)\right)\right)\right]^{2} .\right.
$$

We parametrize the space of hyperplanes by their implicit equations. Thus, every vector $\nu \in[-1,1]^{5} \backslash\{0\}$ represents an hyperplane and two such vectors represent the same hypeprplan if and only if their coordinates are proportional. Notice that problem (19) is of course not convex but has a few parameters. Thus, it is possible to obtain a reasonably good approximation of an optimal $H$ by a global direct 


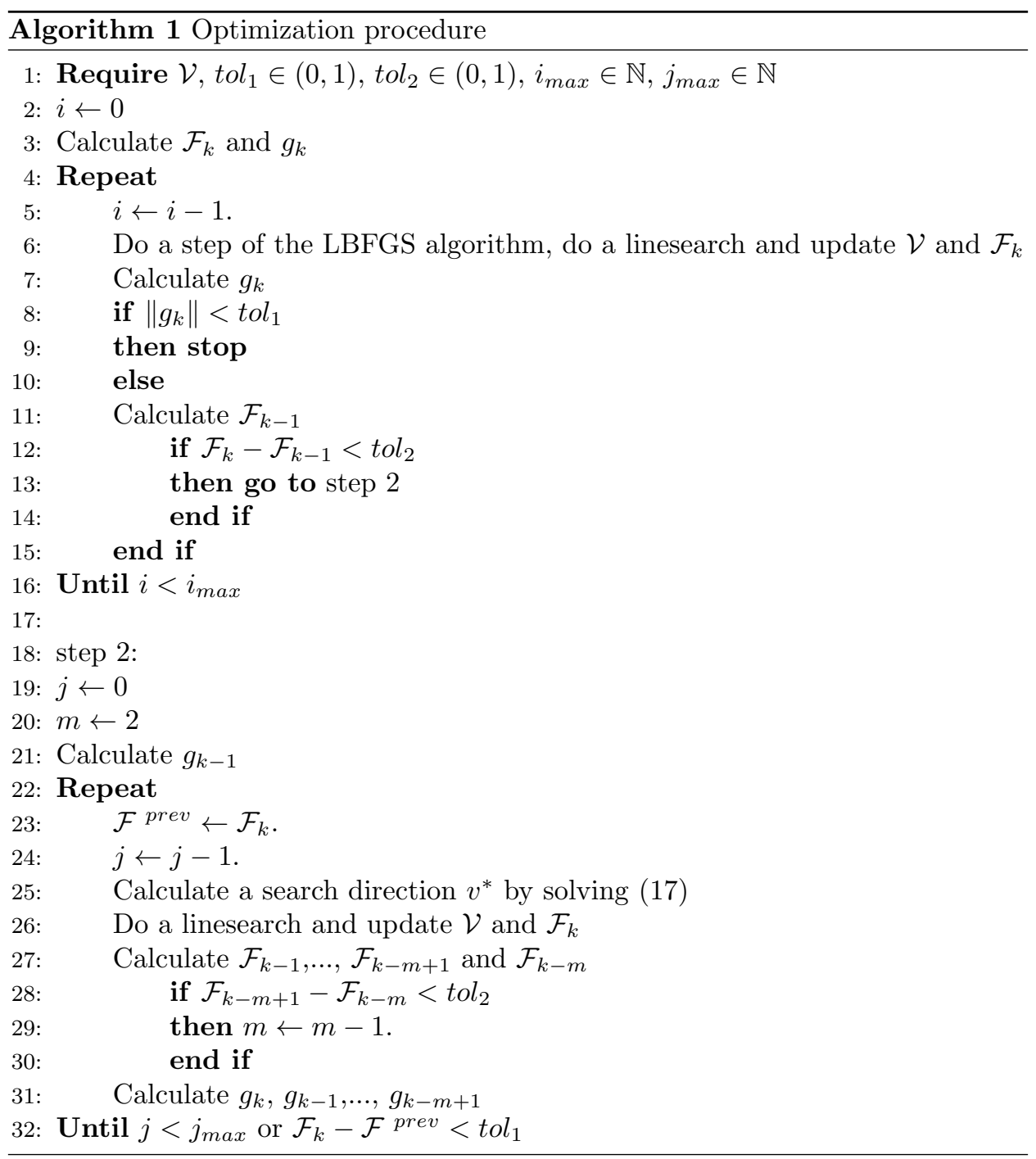

search algorithm which only requires cost function evaluations. In the four test cases under consideration below, it has been possible to obtain a first hyperplane $H$ which is an approximated symmetric hyperplane for the set $\Omega$ up to a precision of 0.01 for 1000 fixed sampling parameters $\left(\beta_{i}, \theta_{i}, \phi_{i}\right)$. This means that every term in the sum (19) is smaller than 0.01. Once a first hyperplane $H_{1}$ (described by $\nu_{1}$ ) of symmetry has been identified, we tried to complete $\nu_{1}$ into an orthonormal base for which every hyperplane of coordinate is as close as possible from an hyperplane of symmetry. To that purpose, we consider a second optimization problem which is the minimization of $F$ among vectors of $[-1,1]^{5} \backslash\{0\}$ which are orthogonal to $\nu_{1}$. A normalization of that vector defines the second vector or our base. By induction, we define in such a way an orthogonal basis which has been used to obtain the pictures of figures 5 and 6 . More precisely, we first rotated every computed shape with respect to its associated orthogonal basis. In a second step, we estimated the 
width of the shape in the direction given by the first vector of that basis. Since we always found at least one axis of symmetry in our experiments, this first basis direction is always an axis of symmetry for the computed optimal shape. Finally, we sampled uniformly the width in every four direction of the basis to obtain the orthogonal cuts given in figures 5 and 6 . Every row corresponding to one direction.

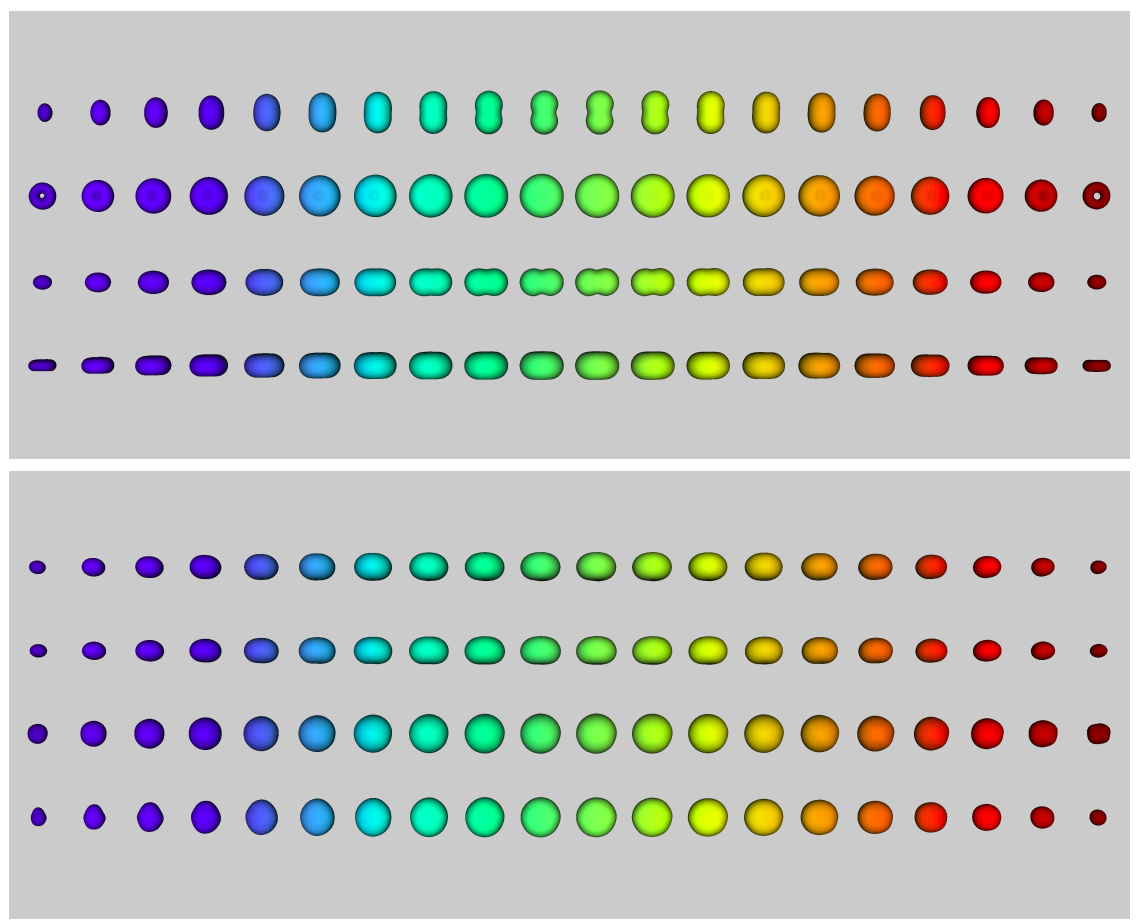

FiguRE 5. Orthogonal cuts of the optimal shapes minimizing the third and the fourth Dirichlet eigenvalues.

\begin{tabular}{|c|c|c|c|}
\hline $\mathrm{i}$ & multiplicity & $\lambda_{i}^{\star}$ & $\lambda_{i}(\mathcal{B})$ \\
\hline 3 & 2 & $\mathbf{5 3 . 9 5}$ & 56.50 \\
\hline 4 & 3 & $\mathbf{5 7 . 0 6}$ & 58.59 \\
\hline 5 & 4 & $\mathbf{5 8 . 5 9}$ & $\mathbf{5 8 . 5 9}$ \\
\hline 6 & 5 & $\mathbf{6 7 . 0 6}$ & $\mathbf{6 7 . 0 6}$ \\
\hline 7 & 4 & $\mathbf{7 6 . 2 8}$ & 74.57 \\
\hline 8 & 4 & $\mathbf{7 9 . 1 7}$ & 81.39 \\
\hline
\end{tabular}

TABLE 1. Optimal values for $\lambda_{i}^{*}$ and the corresponding multiplicity.

\section{Discussion OF OUR NUMERICAL RESUlts}

The minimizers of $\lambda_{1}$ and $\lambda_{2}$ are known to be (respectively) a ball and two balls of the same volume (cf. $[24,34,44,35]$ ). We considered the minimization of $\lambda_{i}$, for 

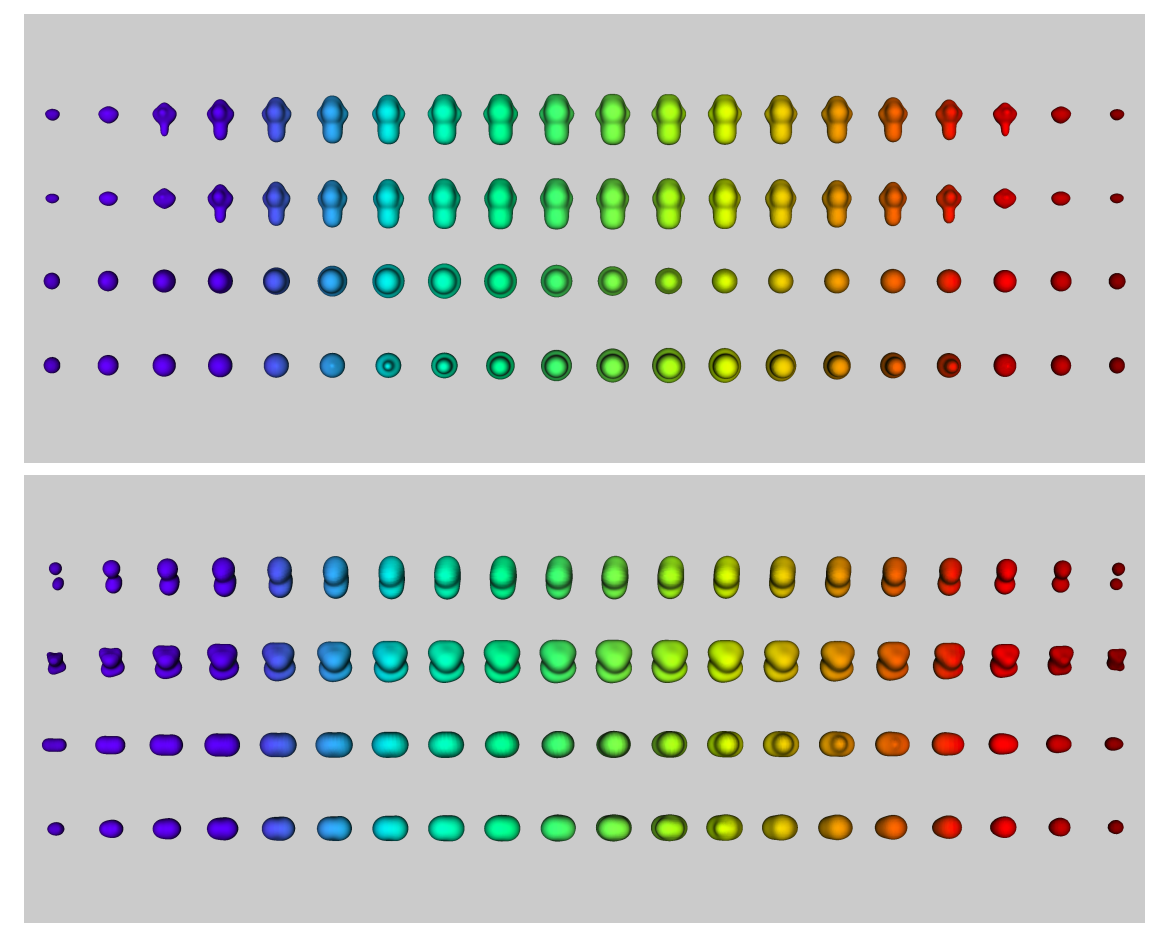

Figure 6. Orthogonal cuts of the optimal shapes minimizing the $7^{\text {th }}$ and $8^{\text {th }}$ Dirichlet eigenvalues.

$i=3,4, \ldots, 8$. The results are summarized in Table 1 . The last column shows the best results obtained for unions of balls. The results presented in Table 1 show that our numerical optimizer for $\lambda_{3}$ has smaller third eigenvalue than the union of three identical balls, which is the best union of balls for this eigenvalue. These numerical estimates provide a counterexample to the Conjecture 'Open Problem 8' in [31]. Our results also suggest that the minimizer for $\lambda_{4}$ is a nontrivial connected domain, while the fifth eigenvalue is minimized by the ball and the sixth is minimized by a union of two balls of different volumes, in such a way that the first eigenvalue of the smallest ball is equal to the fifth eigenvalue of the largest ball. To conclude this numerical study, let us point out a striking fact: as in the three dimensional case, cuts of optimal profile in four dimension display a strong similarity with optimal domains in lower dimension. For instance, the computed optimal shape for $\lambda_{3}$ in $4 \mathrm{D}$ has cuts (see figure 5) which look very close from the optimal shape which is suspected to minimize $\lambda_{3}$ under volume constraint in three dimension (see the left picture in figure 7). Analogously, some cuts of the computed optimal shape for $\lambda_{8}$ in $4 \mathrm{D}$ have a profile very similar to the $3 \mathrm{D}$ optimal shape associated to $6^{\text {th }}$ eigenvalue (see the right picture in figure 7). We think that a better understanding of these dimensional correlations would lead to deep and new results in the area.

\section{REFERENCES}

[1] V. Akçelik, L.-Q. Lee, Z. Li, C. Ng, L. Xiao and K. Ko, Large scale shape optimization for accelerator cavities, Journal of Physics: Conference Series 180, (2009), 012001. 

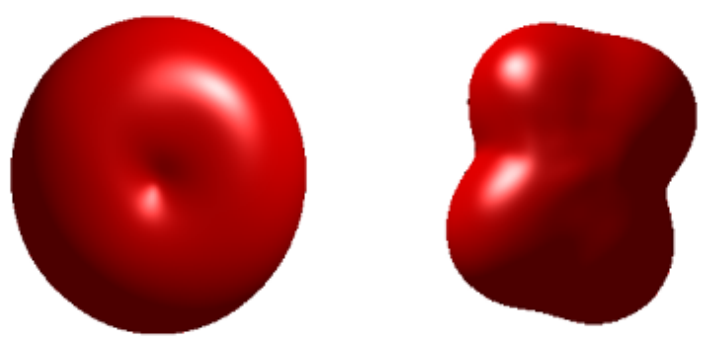

Figure 7. Computed optimal shape associated to the third (left) and the $6^{\text {th }}$ eigenvalue (right) of the Dirichlet laplacian in dimension 3 under measure constraint.

[2] G. Allaire, S. Aubry, and F. Jouve, Eigenfrequency optimization in optimal design, Comput. Methods Appl. Mech. Engrg., 190 (2001), 3565-3579.

[3] C.J.S. Alves, On the choice of source points in the method of fundamental solutions, Eng. Analysis with Bound. Elements, 33 (12), (2009), 1348-1361.

[4] C.J.S. Alves and P.R.S. Antunes, The Method of Fundamental Solutions applied to the calculation of eigenfrequencies and eigenmodes of $2 \mathrm{D}$ simply connected shapes, Computers, Materials \& Continua 2, (4) (2005), 251-266.

[5] C.J.S. Alves and P.R.S. Antunes, The Method of Fundamental Solutions applied to some inverse eigenproblems, SIAM J. Sci. Comput. 35(3), (2013), A1689-A1708.

[6] P. R. S. Antunes and P. Freitas, Numerical optimization of low eigenvalues of the Dirichlet and Neumann Laplacians, J. Opt. Theor. Appl. 154 (2012), 235-257.

[7] P. R. S. Antunes, Numerical calculation of eigensolutions of 3D shapes using the method of fundamental solutions, Numer. Methods Partial Differential Equations, 27 (2011), $1525-1550$.

[8] P. R. S. Antunes, Is is possible to tune a drum?, submitted.

[9] V. I. Arnold, Mathematical Methods of Classical Mechanics, Springer, New York, (1978).

[10] A. H. Barnett and T. Betcke, Stability and convergence of the method of fundamental solutions for Helmholtz problems on analytic domains, J. Comput. Phys. 227, (2008), $7003-7026$.

[11] E. Berchio, A. Ferrero and F. Gazzola, Structural instability of nonlinear plates modelling suspension bridges: mathematical answers to some long-standing questions, Nonlin. Anal. Real World Appl. 28 (2016), 91-125.

[12] M. V. Berry and C. J. Howls, High orders of the Weyl expansion for quantum billiards: resurgence of periodic orbits, and the Stokes phenomenon, Proc. Roy. Soc. A 447 (1994), $527-555$.

[13] T. Betcke and L. N. Trefethen, Reviving the method of particular solutions, SIAM Rev., 47 (2005), 469-491.

[14] D. Bucur, Minimization of the $k$-th eigenvalue of the Dirichlet Laplacian, Arch. Ration. Mech. Anal. 206 (2012), 1073-1083.

[15] D. Bucur and A. Henrot, Minimization of the third eigenvalue of the Dirichlet Laplacian, Proc. Roy. Soc. London 456 (2000), 985-996.

[16] J. T. Chen, I. L. Chen, and Y. T. Lee, Eigensolutions of multiply connected membranes using the method of fundamental solutions, Engrg. Anal. Boundary Elements, 29 (2005), $166-174$.

[17] J. H. Conway and N. J. A. Sloane, Sphere Packings, Lattices and Groups, 2nd ed., New York: Springer-Verlag (1993).

[18] R. Courant and D. Hilbert, Methods of Mathematical Physics, Vol. I, Interscience Publishers, New York (1953).

[19] S. J. Cox and P. X. Uhlig, Where best to hold a drum fast, SIAM Rev. 45(1) (2003), $75-92$. 
[20] L. Danzer, Finite point-sets on $S^{2}$ with minimum distance as large as possible, Discr. Math., 60 (1986), 3-66.

[21] S. K. Donaldson, Self-dual connections and the topology of smooth 4-manifolds. Bull. Amer. Math. Soc. 8 (1983), 81-83.

[22] T. Erber and G. M. Hockney, Equilibrium configurations of N equal charges on a sphere, J. Phys. A: Math. Gen. 24 (1991), L1369-L1377.

[23] T. Erber and G. M. Hockney, Comment on "Method of constrained global optimization," Phys. Rev. Lett. 74 (1995), 1482.

[24] G. Faber, Beweis, dass unter allen homogenen membranen von gleicher flüche und gleicher spannung die kreisfürmige den tiefsten grundton gibt, Sitz. ber. bayer. Akad. Wiss. (1923), 169-172.

[25] L. Fejes Tóth, Über die Abschätzung des kürzesten Abstandes zweier Punkte eines auf einer Kugelfläche liegenden Punktsystems, Jber. Deutch. Math. Verein. 53 (1943), 66-68.

[26] M.H. Freedman (1982), The topology of four-dimensional manifolds, Journal of Differential Geometry 17 (3): (1982), 357-453.

[27] L. Glasser and A. G. Every, Energies and spacings of point charges on a sphere, J. Phys. A: Math. Gen. 25 (1992), 2473-2482.

[28] F. de Gournay, Velocity extension for the level-set method and multiple eigenvalues in shape optimization. SIAM journal on Control and Optimization, 45(1) (2006), 343-367.

[29] E. Haber, A multilevel level-set method for optimizing eigenvalues in shape design problems, J. Comp. Phys. 198 (2004), 518-34.

[30] L. Henrique, J. Antunes, J. S. Carvalho, Shape optimization techniques for musical instrument design, J. Acoust. Soc. Am. 110, (2001) 2648.

[31] A. Henrot, Extremum problems for eigenvalues of elliptic operators, Frontiers in Mathematics. Birkhäuser Verlag, Basel, (2006).

[32] A. Henrot and E. Oudet, Minimizing the second eigenvalue of the Laplace operator with Dirichlet boundary conditions, Arch. Ration. Mech. Anal. 169 (2003), 73-87.

[33] M. Katsurada, A mathematical study of the charge simulation method. II, J. Fac. Sci. Univ. Tokyo Sect. IA Math. 36 (1) (1989), 135-162.

[34] E. Krahn, Über eine von Rayleigh formulierte minimaleigenschaft des kreises, Math. Annalen 94 (1924), 97-100.

[35] E. Krahn, Über Minimaleigenshaften der Kugel in drei und mehr Dimensionen, Acta Comm. Univ. Dorpat. A9 (1926), 1-44.

[36] D. C. Liu and J. Nocedal, On the Limited Memory Method for Large Scale Optimization. Mathematical Programming B. 45 (3) (1989): 503-528.

[37] D. Mazzoleni and A. Pratelli, Existence of minimizers for spectral problems, Journal de Mathématiques Pures et Appliquées, 100(3) (2013), 433-453.

[38] P.M. Morse and H. Feshbach, Methods of Theoretical Physics, Part I. New York: McGraw-Hill, (1953), 623-624.

[39] O. R. Musin and A. S. Tarasov, The Tammes Problem for N = 14. Exp. Math. 24, (2015) $460-468$.

[40] D. Noreland, R. Udawalpola, and M. Berggren, A hybrid scheme for bore design optimization of a brass instrument, J. Acoust. Soc. Am., 128(3) (2010), 1391-1400.

[41] E. Oudet, Numerical minimization of eigenmodes of a membrane with respect to the domain, ESAIM Control Optim. Calc. Var. 10 (2004), 315-330.

[42] S.J. Osher and F. Santosa, Level set methods for optimization problems involving geometry and constraints. 1: Frequencies of a two-density inhomogeneous drum, Journal of Computational Physics 171(1) (2001), 272-288.

[43] B. Osting and C.-Y. Kao, Minimal convex combinations of sequential Laplace-Dirichlet eigenvalues, SIAM J. Sci. Comput, 35(3) (2013), B731-B750.

[44] G. Pólya, On the characteristic frequencies of a symmetric membrane, Math. Zeit. 63 (1955), 331-337.

[45] B. Rousselet and D. Chenais, Continuité et différentiabilité d' élements propres: Application à l'optimisation de structures. Appl. Math. and Optim., 22, (1990), 27-59.

[46] R.M.L. Tammes, On the origin number and arrangement of the places of exits on the surface of pollengrains, Rec. Trv. Bot. Neerl. 27 (1930), 1-84.

[47] N. J. A. Sloane, Spherical codes: Nice arrangements of points on a sphere in various dimensions. Available at http://neilsloane.com/packings/index.html\#I 
[48] C.H. Taubes, (1987), Gauge theory on asymptotically periodic 4-manifolds., Journal of Differential Geometry 25, (1987), 363-430,

[49] S.A. Wolf and J.B. Keller, Range of the first two eigenvalues of the Laplacian. Proc. R. Soc. Lond. Ser. A, Math. Phys. Sci. 447, (1994), 397-412.

Group of Mathematical Physics, Faculdade de Ciências da Universidade de Lisboa, Campo Grande, Edifício C6 1749-016 Lisboa, Portugal

E-mail address: prantunes@fc.ul.pt

LJK, Université de Grenoble, 51 Rue des Mathématiques, F-38041 Grenoble Cedex 9

E-mail address: edouard.oudet@imag.fr 